\title{
Giant incidentally-detected non-functional adrenal myelolipoma - a case report
}

Alina Mihaela PASCU, Mircea Daniel HOGEA, Lorena DIMA, Petru Iulian IFTENI, Claudia GAVRIS,, Marius Alexandru MOGA



FACULTY OF MEDICINE, TRANSILVANIA UNIVERSITY OF BRAȘOV, ROMANIA

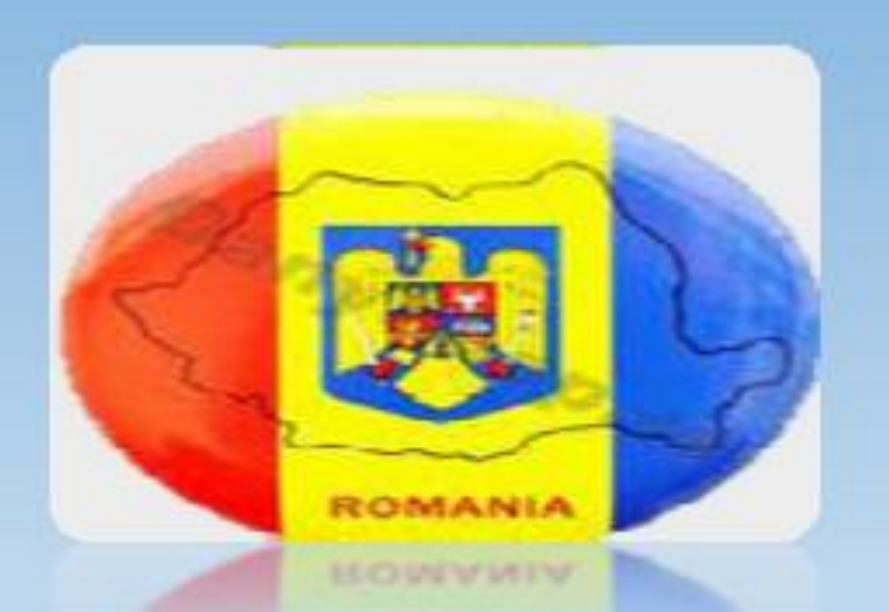

Introduction

Adrenal myelolipoma is a rare benign tumor composed of mature adipose tissue and haematopoietic elements developed within the adrenal gland, usually unilateral, asymptomatic, and non-functional. Their real incidence is unknown.

\section{Case report}

A 56 year-old female was admitted to our surgery department for abdominal distension and the presence of a large palpable tumor in the right abdomen discovered during an abdominal ultrasonographic check-up for an incisional hernia, and with no noticeable symptoms.

Patient's medical history: a 3rd stage systemic arterial hypertension, insulin-requiring type 2 diabetes mellitus, hysterectomy with anexectomy for a cervical cancer.

The abdominal ultrasound examination showed a large hyperechogenic tumor, heterogenic, located in the right flank and hypochondriac region.

The contrast enhanced computed tomography scan revealed a large retroperitoneal tumor $(25 \times 17 \times 21 \mathrm{~cm})$ well delimitated with a heterogenic structure (liquid and non-iodophile lipid densities, and central calcifications). The mass was in contact with the right hepatic lobe, inferior vena cava, ascendant and transverse colon, and, posteriorly, with the diaphragm (Figure 1). The CT also showed a $74 \mathrm{~mm}$ incisional hernia with incarcerated small bowel.

Biochemical evaluation: serum potassium $=4.2 \mathrm{mmol} / \mathrm{L}$, serum cortisol $=204.8 \mathrm{nmol} / \mathrm{L}$; after a $1-\mathrm{mg}$ overnight dexamethasone suppression test serum cortisol $=22.1 \mathrm{nmol} / \mathrm{L}$.

Taking into account the tumoral mass dimensions, surgical resection was decided. Because of the medial incisional hernia, a medial subxifoid-pubic incision was performed. The mass was dissected from the upper pole of the right kidney, from the right hepatic lobe and from the inferior vena cava and was sent for histological evaluation (Figure 2).

The microscopic examination: adrenal cortex, adipose tissue, and multiple (extramedullary) haematopoiesis sites adrenal myelolipoma (Figure 3).

The patient had a good postoperative evolution.

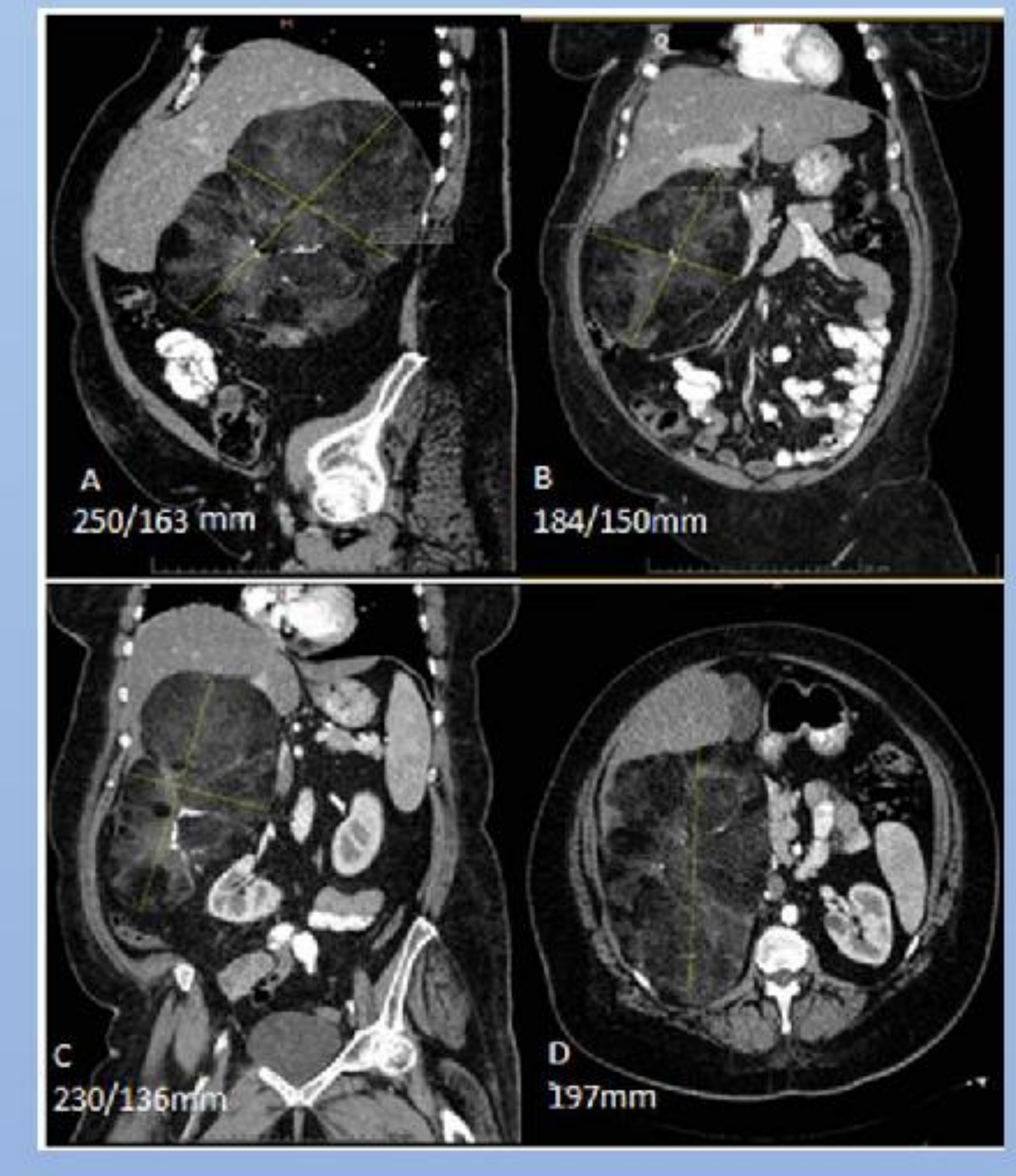

Figure 1. CT aspect of the intra-abdominal mass

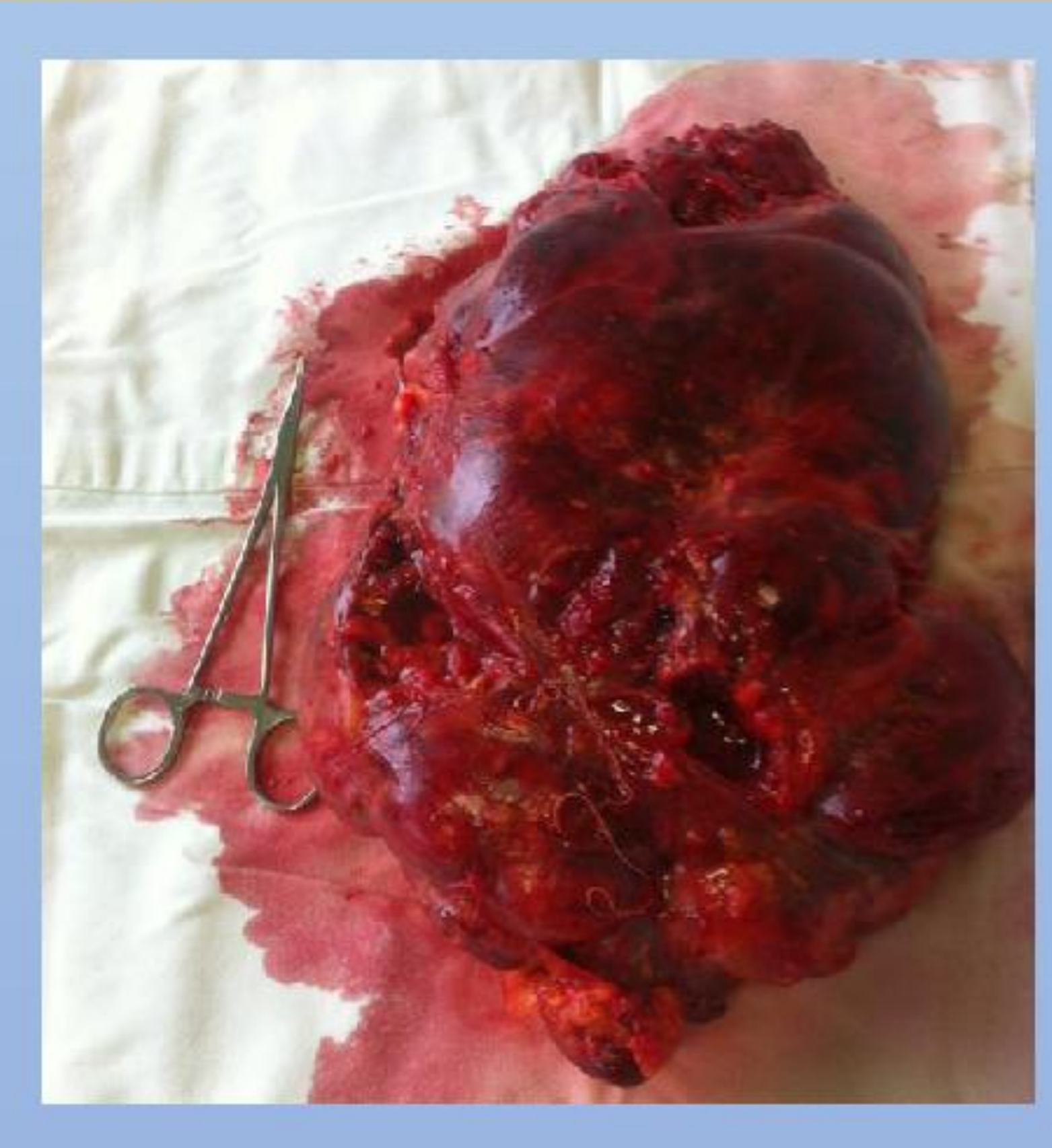

Figure 2. Macroscopic aspect of the tumor

\section{Conclusions}

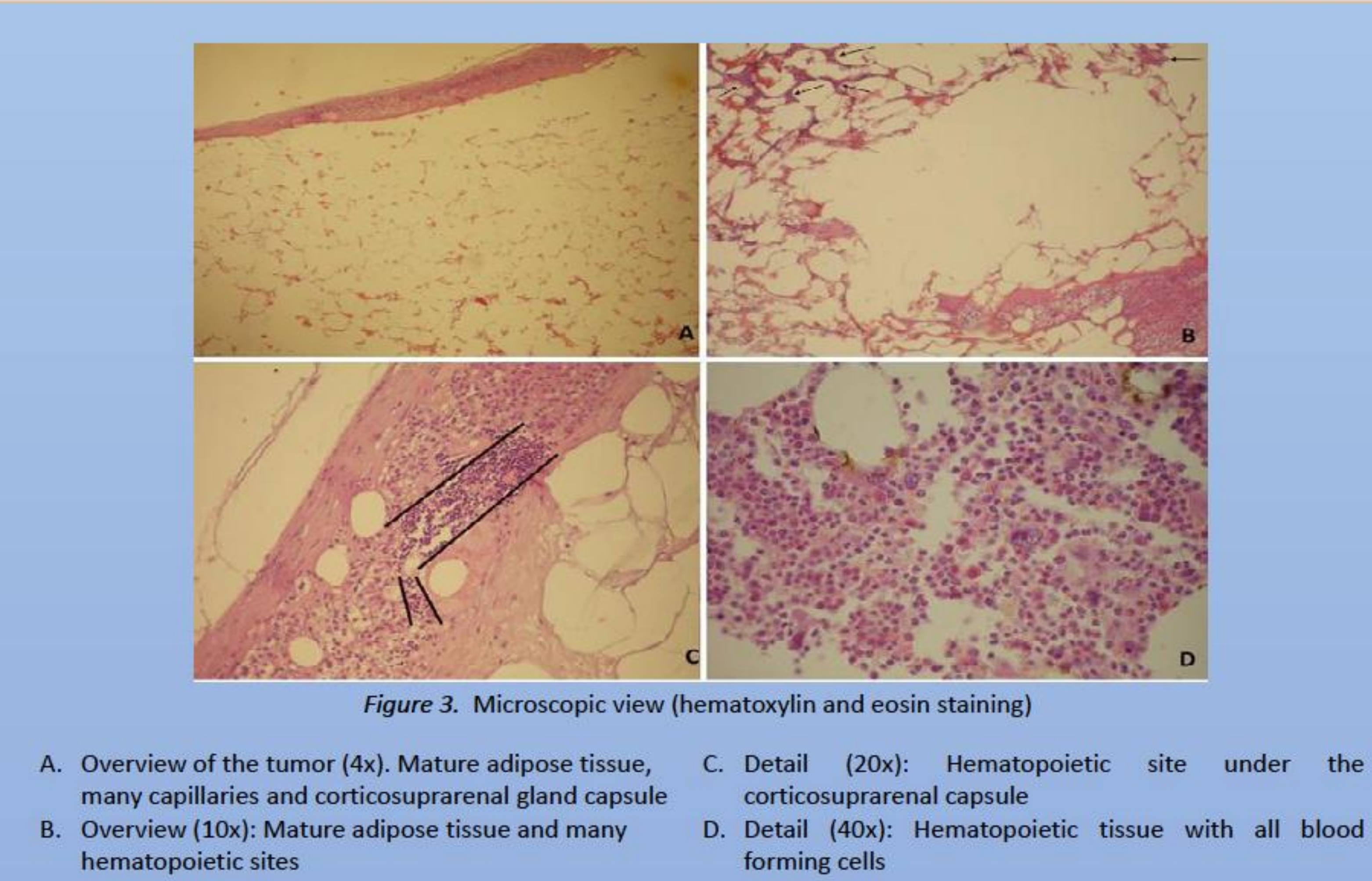

B. Overview (10x): Mature adipose tissue and many
hematopoietic sites

The incidentally detected tumoral mass in the clinical condition of the patient challenged to differentiate an adrenal adenoma (functional or not) from a retroperitoneal lipoma/liposarcoma, or metastasis. A particularity of this myelolipoma was the tumor size (approx. $8.925 \mathrm{~cm}^{3}$ ).

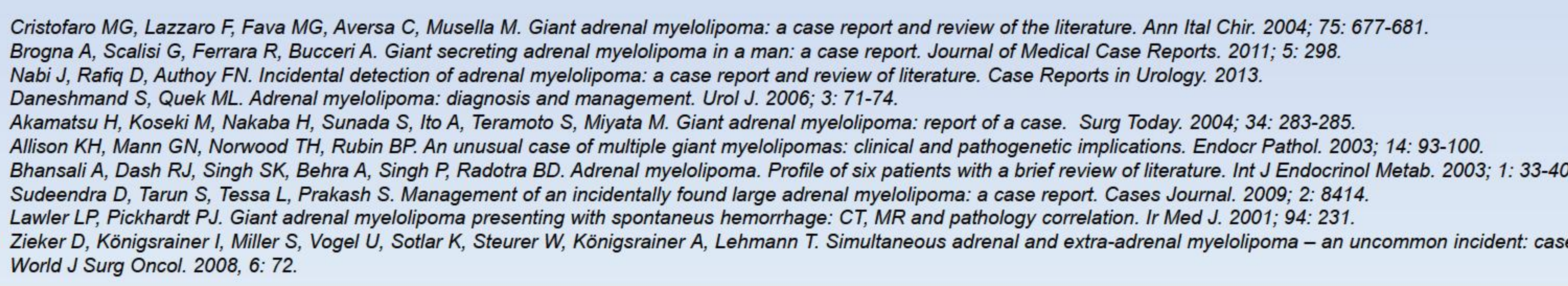

